\title{
Light activation of Orange Carotenoid Protein reveals initial C8'-C7' double bond trans/cis photoisomerization
}

V.U. Chukhutsina*, J. Baxter*, A. Fadini*, R.M. Morgan*, M.A. Pope*, K. Maghlaoui*, C. Orr\#, A.Wagner\#, J.J. van Thor*

*Department of Life Sciences, Imperial College London, London SW7 2AZ, United Kingdom \# Diamond Light Source, Harwell Science and Innovation Campus, Didcot OX11 0DE, United Kingdom

\section{Summary}

Orange Carotenoid protein (OCP) is the only known photoreceptor which uses carotenoid for its activation. It is found exclusively in cyanobacteria, where it functions to control lightharvesting of the photosynthetic machinery. The mechanism of photoactivation includes $\mathrm{H}$ bond rupture between the carotenoid keto group and the protein moiety in the C-terminal domain $(\mathrm{CTD})^{1-4}$ but details of the primary photochemical reactions and structural dynamics are not yet resolved. Here we present data from second-to-minute time-resolved crystallography under bright illuminations capturing the primary photoproduct and structure of subsequent reaction intermediates. The initial photoproduct shows carotenoid trans/cis photoisomerization at the C7'-C8'double bond and structural changes in the N-terminal domain with minute timescale kinetics. These are followed by a thermally-driven cis/trans isomerization that recovers to the dark state carotenoid configuration. In addition, the structural changes are propagated to the CTD resulting in the eventual H-bond rupture. The photoisomerization and its transient nature are confirmed in OCP crystals and solutions by FTIR and UV/VIS spectroscopy. This study reveals the photoisomerization of the carotenoid and subsequent thermal structural reactions as the basis primary events of OCP photoreception. It is an initial step in understanding and potentially controlling OCP dynamics, offering the prospect of novel applications in biomass engineering as well as advancing optogenetics and bioimaging.

\section{Main}

Carotenoids, found in plants, algae and microorganisms constitute the most widely spread group of pigments 5 . Carotenoids are isoprenoids made-up of a central carbon chain of alternating single and double bonds with unique photochemical properties. The S0-S1 electronic transition is optically forbidden in carotenoids and light absorption occurs through the intense S0-S2 transition ${ }^{6}$ which is responsible for their yellow, orange and red colours. After light absorption, the $\mathrm{S} 1$ dark state is populated through internal conversion, after which the excitation decays with an ultrashort (ps) lifetime ${ }^{7}$. The structure and conformation of carotenoids determine the energy landscape of their electronic states and, as a result, their biological functions. Carotenoid chromophores function as key photosynthetic regulators of biomass production $^{8,9}$ when their S1 states act as efficient quenchers of chlorophyll excited states $^{10,11}$. Furthermore, carotenoid excited states also act as energy donors in photosynthetic light-harvesting ${ }^{7}$ as well as photoreceptors in light-signalling ${ }^{12}$. The structural and conformational variability of carotenoids which underlie their diverse biological functions remains to be better understood. The canthaxanthin (CAN) carotenoid bound to the Orange 
Carotenoid Protein is responsible for a light signalling function. The OCP undergoes a photocycle ${ }^{13}$ in which the keto-carotenoid also plays several different roles: in the inactive state (orange, $\mathrm{OCP}^{\mathrm{O}}$, Fig 1a), it senses blue-green light to initiate the photocycle, resulting in a final product which has undergone substantial structural conformational changes and exhibits distinct separation of the two OCP domains, namely, the all-helical N-terminal domain (NTD) and an $\alpha / \beta$-fold $\mathrm{C}$-terminal domain (CTD) ${ }^{14}$. In the active state, following $\mathrm{OCP}^{\mathrm{R}}$ (red state) binding to the cyanobacterial light-harvesting antenna, the keto-carotenoid dissipates excess absorbed light energy, a vital process to cyanobacterial survival in turbulent waters ${ }^{12,15}$.

The mechanism and primary photochemical reaction of the keto-carotenoid that triggers this $\mathrm{OCP}^{\mathrm{O}}-\mathrm{OCP}^{\mathrm{R}}$ conversion is currently unknown and requires structural evidence ${ }^{12,15}$. It has been suggested that OCP photoactivation is initiated by H-bond rupture between the 4-keto group of the $\beta 1$-ring and neighbouring residues Y201 and W288. ${ }^{1-4,16-18}$ Several proposals have been made for the primary photochemistry resulting in this particular H-bond rupture: (1) a charge transfer state ${ }^{19}$; (2) a shift in keto-enol equilibrium ${ }^{2}$; (3) a transfer of vibrational energy from the carotenoid to the protein leading to the H-bond rupture ${ }^{20}$, similarly to H-bond rupture in bacteriorhodopsin ${ }^{21}$; (4) a structurally distorted form of the carotenoid in the S1 state characterized by increased carotenoid planarity ${ }^{3}$; (5) a photoinduced s-isomerization of the C6-C7 single bond, flipping the orientation of the $\beta 1$-ring by $90^{\circ 1}$. Support for s-isomerization isomerization in OCP stemmed from the X-ray structure of the isolated NTD (also known as red carotenoid protein, $\mathrm{RCP}$ ), in which the keto-carotenoid was present in a $\mathrm{C} 6-\mathrm{C} 7 \mathrm{~s}$-cis conformation $^{1}$, and frequency calculations showing agreement between a $\mathrm{C} 6-\mathrm{C} 7$ s-cis conformation and $\mathrm{OCP}^{\mathrm{R}}$ Resonance Raman data ${ }^{22}$. However, the hypothesis for s-isomerization in $\mathrm{OCP}^{\mathrm{R}}$ is not consistent with recent infra-red (IR) anisotropy observations ${ }^{3}$. A recent study of the fluorescence spectra and yields of $\mathrm{OCP}^{\mathrm{O}}$ with different carotenoids suggested that the mechanism of OCP photoactivation does not involve the keto group of the $\beta 1$ ring but includes out-of-plane motions of the $\beta 2$ ring $^{22}$. To date, details of the primary photochemical reactions of the carotenoid that trigger OCP light-activation remain elusive and are yet to be unambiguously resolved.

Figure 1c shows the post-illumination absorption changes measured in crystals and solutions of OCP confirming that the photoactivation reactions proceed the same way._In contrast to a previous crystallographic study investigation of OCP photoactivation ${ }^{2}$, we observe that the absorbance spectra of $\mathrm{OCP}^{\mathrm{O}}$ in solution and in crystals are nearly identical (Fig 1b), thereby providing evidence for the same electronic structure of the carotenoid in both states. Illumination of crystals with $410-\mathrm{nm}$ light and $3.5 \mathrm{~mW} \mathrm{~cm} \mathrm{~m}^{-2}$ power density causes a significant spectral red-shift of $25 \mathrm{~nm}$ due to $\mathrm{OCP}^{\mathrm{O}}-\mathrm{OCP}^{\mathrm{R}}$ conversion within 5 min-on average (Fig $1 \mathrm{~b}$ insert) similar to the shift observed in solution (Fig 3). Since OCP crystals display a photoresponse to the same extent as that observed in solution, we can confidently infer that X-ray crystallography is well suited to investigate the process of OCP light-activation and in particular the early photochemical reaction and structural response in the photocycle.

Early reaction intermediates were captured by intense $\mathrm{CW}$ illumination and paired with cryo-trapping according to the overall rate kinetics determined from spectroscopy. The refined dark structure $\left(\mathrm{OCP}_{\text {Dark }}\right.$, Fig 1a) is in good agreement with those reported in previous X-ray studies $^{1,2,13,16}$ showing a similar carotenoid conformation (Supplementary Table 1). Together 
with the protein and the CAN carotenoid structures, we have identified the location of glycerol, acetate, $\mathrm{Cl}^{-}$and 440 water molecules. In addition to $\mathrm{OCP}_{\text {Dark }}$, the X-ray data in the four illuminated structural intermediates I1, I2, I3 and I4 corresponding to $1 \mathrm{~min}, 2 \mathrm{~min}, 5 \mathrm{~min}$ and $10 \mathrm{~min}$ of illumination were collected and structures solved at comparable resolution and statistics. Q-weighted $\mathrm{F}_{\text {Light }}-\mathrm{F}_{\text {Dark }}$ difference electron density (DED) maps ${ }^{23}$ and coordinates refined to extrapolated structure factors (see Materials and Methods, Fig. S8-S9) for CAN are presented in Fig 1c. For the initial step of OCP light activation we observe isomerization of CAN around the C8'-C7' double bond (Fig $1 \mathrm{c}-\mathrm{d}$ ). The above isomerization is also detected in $\mathrm{OCP}_{\mathrm{I} 1}$ and $\mathrm{OCP}_{\mathrm{I} 2}$, though less pronounced in the latter, and fully disappears after 5 min in $\mathrm{OCP}_{\mathrm{I} 3}$ and $\mathrm{OCP}_{\mathrm{I} 4}$ judging from DED, $2 \mathrm{mFo}-\mathrm{DFc}$ maps and refined coordinates using extrapolated structure factors (Fig 1, Supplementary Table 1).

Therefore, the CAN carotenoid converts from an all-trans to a C8'-C7'-cis state with complete accumulation of the photoproduct within 1 min of illumination at the experimental light intensity used. UV-VIS spectroscopy confirms that the rate of accumulation of the primary product is proportional to the illumination intensity (Supplementary Fig 10). Complete thermal relaxation of C8'-C7'-cis CAN back to all-trans state occurs after a 5 min illumination (Supplementary note 9 and Fig 10). In contrast to the current prevailing model ${ }^{1-4,16-18}$ favouring a H-bond rupture in CTD as the primary photoproduct of the OCP photocycle, our $\mathrm{X}$-ray data directly demonstrate that a transient C8'-C7'-cis state is generated. The findings redefine the molecular light-activation mechanism of OCP and reveal, for the first time, details of its primary photochemical reaction. To our knowledge, there has been no reported case of double bond photoisomerization of the carotenoid carbon chain regulating enzymatic activity or the biological function of a protein.

Photoinduced structural changes occur throughout the OCP protein and propagate in a time-dependent manner. The $\mathrm{F}_{\mathrm{II}}-\mathrm{F}_{\mathrm{Dark}}$ map reproducibly displays differences in three regions (Fig 2, Supplementary Fig 6a, Supplementary Fig 8): in the NTD with the highest DED amplitudes within $6 \AA$ from the CAN $\beta 2$ ring (Zone 1, Fig 1c), in the N-terminal extension (Zone 2, Fig 1c) and at the interface between the two domains. The DED signals appear in the CTD (Zone 3, Fig 1c, Supplementary Fig 8) with time delays over 1 min. In the I2 intermediate (i.e. after 2 min of illumination), a DED map appears primarily in the region of the CAN binding pocket before later moving along helices of the CTD (I3, I4, Fig 1c). Concomitant with the above, the DED signals in Zones 1 and 2 disappear in I3 and I4. These results indicate that NTD is the primary site of action in the OCP light-activation. Therefore, the onset of photosensing in the OCP photocycle involves structural changes in the NTD but not in the CTD as previously suggested ${ }^{2}$.

The structural rearrangements in the intermediates I1, I2, I3 and I4 were further analysed from the crystallographic data. Tyrosine Y44 and Tryptophan W110 are located in closest proximity ( $4 \AA$ ) to the C7'-C8' bond in the hydrophobic pocket which also contains the $\beta 2$-ring of the carotenoid (Fig 3 a). Together with W41, residues Y44 and W110 non-covalently bind the carotenoid $\beta 2$-ring through $\pi-\pi$ stacking interactions. ${ }^{16}$ We do not observe any substantial movement of $\mathrm{W} 110$ in $\mathrm{OCP}_{\mathrm{I} 1}$ as compared to $\mathrm{OCP}_{\text {Dark }}$ but, instead, notice a $31^{\circ}$ turn of the Y44 sidechain (Fig 3 a). This observation suggests that Y44 acts as a mediator of C7'C8' isomerization to trigger structural changes in NTD. The structures of I1 and I2 
intermediates suggest that the flip of the Y44 sidechain causes the displacement of many amino acids in NTD. These include residues Y111 and G114, which are closest to Y44 and are rearranged in $\mathrm{OCP}_{\mathrm{I} 1}$ and $\mathrm{OCP}_{\mathrm{I} 2}$ (Fig 3a). In agreement with our crystallographic observations, previous spectroscopic analyses demonstrated that the mutation of Y44 to a serine totally inhibits $\mathrm{OCP}^{\mathrm{O}}-\mathrm{OCP}^{\mathrm{R}}$ conversion ${ }^{18}$. As a result, substantial charge-density redistribution around the $\beta 2$-ring is expected in $\mathrm{OCP}_{\mathrm{I} 1}$ (Supplementary Fig 11). These induced rearrangements are transient and the amino acids move back to their original location in $\mathrm{OCP}_{\text {Dark }}$ after 5-10 min (Fig 3 a). Leucine L37, which has been proposed to play an important role in OCP lightactivation ${ }^{22}$, does not undergo any significant movement upon illumination (Supplementary Fig 13). We therefore conclude that C7' $\mathrm{C} 8$ ' isomerization causes rearrangement of the CAN 32-ring binding pocket in NTD which is most likely mediated by a contact involving Y44.

The rearrangement of the $\beta 2$-ring binding pocket coincides with further conformational changes within the NTE and the NTD, affecting primarily helices $\alpha \mathrm{A}-\alpha \mathrm{C}$ and $\alpha \mathrm{E}-\alpha \mathrm{H}$ as can be judged from the location of DED signals-(Fig 2). The amplitude of these signals is highest after a 1 min illumination $\left(\mathrm{F}_{\mathrm{II}}-\mathrm{F}_{\mathrm{Dark}}\right)$ followed by a decrease after a 2 min illumination $\left(\mathrm{F}_{\mathrm{I} 3}-\right.$ $\left.F_{\text {Dark }}\right)$ and complete disappearance after a $10 \mathrm{~min}$ illumination $\left(\mathrm{F}_{\mathrm{I}}-\mathrm{F}_{\text {Dark }}\right)$. The further conformational rearrangements of the $\alpha A-\alpha C$ and $\alpha E-\alpha H$ affect the hydrogen-bond network by increasing the number of polar contacts in the main backbone of $\mathrm{OCP}_{\mathrm{I} 1}$ by $5 \%$ compared to $\mathrm{OCP}_{\text {Dark }}$. As a result, 14 new H-bonds are created in the backbone of $\mathrm{OCP}_{\mathrm{I} 1}$ which gradually disappear in $\mathrm{OCP}_{\mathrm{I} 2}-\mathrm{OCP}_{\mathrm{I} 4}$ (Supplementary Table 5). These results indicate that, following light-activation, the light-sensing process in OCP is hydrogen-bonding mediated since it not only involves the CAN $\beta 2$-ring binding pocket but the whole domain as well.

The DED signals within a $5 \AA$ A radius from the carotenoid at the CTD and NTD interface (Fig 1c) have been assigned to the modification of the salt bridge between residues Arginine R155 and glutamic acid E244 (Fig 4 b) and the reorganisation of water molecules (Fig 3b, Supplementary Fig 12). The salt bridge rupture between R155 and E244 is already observed in $\mathrm{OCP}_{\mathrm{I} 1}$ and it is noticeable as a $1 \AA$ distance increase between residues E244 and R155 (Fig 3b). The position of R155 is modulated by the assigned water molecules $\mathrm{HOH} 41$ and $\mathrm{HOH} 46$ through H-bonding (Fig 3b, Supplementary Fig 13). The positions of $\mathrm{HOH} 41, \mathrm{HOH} 46, \mathrm{Cl}^{-}$ anion and other water molecules in the water channel $\mathrm{s}$ are rearranged during the entire illumination period (Supplementary Fig 12, Supplementary Fig S14, Fig 3b). In the final illumination product $\left(\mathrm{OCP}_{\mathrm{I}}\right.$, Fig $\left.3 \mathrm{~b}\right)$ the distance between residues E244 and R155 is restored to the original $3 \AA$, suggesting that, although an important event in the initial steps of light activation, salt-bridge rupture may not be a prerequisite for subsequent domain separation. Previously reported FTIR and XF-MS results suggested that signal propagation of OCP conformational change is mediated by rearrangement of internal hydrogen bonding networks ${ }^{14,24}$. We complement this knowledge by showing that salt bridge rupture between R155 and E244, the important precursor of NTD and CTD separation, occurs in the first illumination step (I1) and is controlled by hydrogen bonding network involving protein-bound water molecules.

In addition we observe an increase in DED signal intensities in the CTD with illumination time (Zone 3, Fig 1). This is first manifested in CTD amino acids P276, L248, P278 and E244 located at the interface of CTD and NTD (Supplementary Fig 13-14). 
The DED signals for CTD (Fig 2) do not represent a complete domain rearrangement but rather a rigid-body motion of the CTD domain in the direction away from the NTD and the dimer interface (Supplementary Fig 14-S15) similar to that previously reported ${ }^{2}$. The increase in distance between W288, Y201 and the keto group of CAN, a suggested prerequisite for H-bond disruption $^{2}$, is only observed at longer illumination times (after 5-10 min, Supplementary Fig 13) and only after rearrangement of the NTD binding pocket. Therefore, the H-bond rupture represents a secondary effect caused by the domains separation and it is not a photo-driven process as previously proposed ${ }^{2,3}$.

Next, we performed FTIR and UV-VIS spectroscopy in solution using the same illumination conditions as for crystals (Figs 1-3) in order to confirm that CAN isomerization is photoinduced (Fig 4) and determine whether the same photoactivation reactions observed in crystals also take place in solution. Fig 4 displays the results from the combined global analysis fits of the UVVIS and FTIR data. Since the experiment was performed under continuous illumination, the Evolution-Associated Spectra (EAS) components do not represent intrinsic lifetimes of the intermediate states but rather dissected population accumulation rates at given illumination power densities (Supplementary Fig $10 \mathrm{a}-\mathrm{b}$ ). Four components of spectral regions (EAS1-4) were identified to describe the absorbance changes in both UV-VIS and IR spectra with lifetimes of $13 \mathrm{~s}, 38 \mathrm{~s}, 162 \mathrm{~s}$ and a steady-state component (infinity). Assuming the same photocycle rates in solution and crystals, the contribution of each component in Figs 1-3 is as follow: (i) I1 state: EAS1 (3\%), EAS2 (40\%) and EAS3 (57\%), (ii) I2 -EAS2 (13\%) and EAS3 (87\%), (iii) I3 -EAS2 (2\%) and EAS3 (98\%) and (iv) I4- EAS3 -100\%. Note that the absence of the final component EAS4 in crystals is probably due to crystal packing constraints on sidechain conformation and dynamicsand it was therefore excluded from the estimates (see the justifications below). The first component (EAS1) exhibits a positive and a negative spectral signature in the visible region (Fig 4a) peaking at $475 \mathrm{~nm}$ and $550 \mathrm{~nm}$ respectively. It does not show significant IR differences (Fig 3b, Supplementary Fig 16). We assign this observation to the conversion of $\mathrm{OCP}^{\circ}$ into a new intermediate species EAS2. EAS2 (at 38s) has a very similar visible spectrum to $\mathrm{OCP}^{\mathrm{O}}\left(\mathrm{t}_{0}\right.$ spectra) though it has an increased absorbance at $350 \mathrm{~nm}$ (Fig 4a insert). The 350-nm band yields the so-called 'cis-peak', a characteristic signature of trans-cis isomerization in carotenoids ${ }^{25,26}$. The IR spectrum of the corresponding EAS2 component in $\mathrm{D}_{2} \mathrm{O}$ buffer shows positive bands in the $1655-1641 \mathrm{~cm}^{-1}$ region while exhibiting a negative band at $1540-1550 \mathrm{~cm}^{-1}(\mathrm{Fig} 4 \mathrm{~b})$. The positive band corresponds to the amide I signal. The position of the 1542-1550 $\mathrm{cm}^{-1}$ bleach is insensitive to $\mathrm{D}_{2} \mathrm{O} / \mathrm{H}_{2} \mathrm{O}$ exchange and agrees with the frequency of $\mathrm{C}=\mathrm{C}$ stretches in canthaxanthin ${ }^{27}$ and other carotenoids ${ }^{28}$. In carotenoids, the oscillator strength of $\mathrm{C}=\mathrm{C}$ stretches is known to decrease with isomerization from all-trans-tocis conformations ${ }^{29}$. Therefore, the presence of the $1542-1550 \mathrm{~cm}^{-1}$ bleach in the EAS2 component agrees very well with its assignment to the trans-cis CAN isomerization as seen on Fig 1c in I1 and indicates that the process is indeed light-induced. The EAS3 component (162s, Fig. 4) confirms that photoisomerization is a transient phenomenon leading to a C7'-C8'trans state in $2.5 \mathrm{~min}$ as can be concluded from a decrease of the $1542 \mathrm{~cm}^{-1}$ bleach. Additional UV-VIS measurement with shorter illumination period confirms that the back C7'-C8' cis-trans relaxation is thermally driven (Supplementary Fig 10 a). To confirm the FTIR assignments, we have performed harmonic frequency calculations at the B3LYP/6-311+G level of CAN in the 
all-trans $\left(\mathrm{CAN}_{\text {Dark }}\right)$ and in the $\mathrm{C}^{\prime}$ '-C8'-cis $\left(\mathrm{CAN}_{\mathrm{II}}\right)$ states using the carotenoid coordinates shown in Fig 2 after optimisation. The major frequency of the $\mathrm{C}=\mathrm{C}$ stretches coupled with the $\mathrm{C}=\mathrm{O}$ stretches in $\mathrm{CAN}_{\text {Dark }}$ is $1542 \mathrm{~cm}^{-1}$, while in the $\mathrm{CAN}_{\mathrm{Il}}$ state, its oscillator strength decreases and redshifts to $1550 \mathrm{~cm}^{-1}$ and the $1542 \mathrm{~cm}^{-1}$ frequency disappears. As a result, the difference signal $\left(\mathrm{CAN}_{\mathrm{I}}-\mathrm{CAN}_{\text {Dark }}\right)$ is characterized by the $1542 \mathrm{~cm}^{-1}$ bleach (Fig $4 \mathrm{c}$ ). Our spectroscopic results, supported by frequency calculations, thus confirm that trans-cis $\mathrm{C} 8$ ' $-\mathrm{C} 7$ ' isomerization is a light-driven process also occurring in OCP in solution.

The EAS2 component also contains positive amide-I FTIR signals peaking at $1651 \mathrm{~cm}^{-}$ ${ }^{1}$ and $1641 \mathrm{~cm}^{-1}$ due to a bleaching signal emerging at later stages (EAS3). These signals, assigned to amide-I peaks based on previous IR signals of OCP WT and mutants ${ }^{3}$, are indicative of $\mathrm{H}$-bond rearrangements which are visible in the $\mathrm{OCP}_{\mathrm{I} 1}$ crystal structure (Supplementary Table 5) and where the positive amide-I FTIR signals can be assigned to the new polar contacts appearing in $\mathrm{OCP}_{\mathrm{I} 1}$ (EAS2, $38 \mathrm{~s}$, Fig 4b). Negative amide-I FTIR signals in the EAS3 component indicate the loss of the newly gained polar contacts as seen in $\mathrm{OCP}_{\mathrm{I} 4}$.

Our observations showing that the absorption at $1651 \mathrm{~cm}^{-1}$ is insensitive to $\mathrm{D}_{2} \mathrm{O} / \mathrm{H}_{2} \mathrm{O}$ exchange, unlike that at $1645 \mathrm{~cm}^{-1}$ (Supplementary Fig 16, Supplemental note 15), is in agreement with previous studies ${ }^{3,24,30}$ and our own crystallographic results (Fig 2).

We have therefore tentatively assigned the $1651 \mathrm{~cm}^{-1}$ and $1641 \mathrm{~cm}^{-1}\left(\mathrm{D}_{2} \mathrm{O}\right) / 1645 \mathrm{~cm}^{-1}$ $\left(\mathrm{H}_{2} \mathrm{O}\right)$ bands to a buried $\alpha$-helix and a solvent exposed $\alpha$-helix of NTD respectively (see Supplementary Discussion 6 for the contribution of water molecules to FTIR data).

Contrary to a previous suggestion ${ }^{24}$, our data does not support any involvement of the C-terminal tail to changes of the $1645 \mathrm{~cm}^{-1}$ peak, since no structural rearrangements are observed in this region in the crystallographic data corresponding to the same time delays (I1I2, Fig 2, Supplementary Fig 8). In line with the final $\mathrm{OCP}_{\mathrm{I} 4}$ crystal structure, where the CTD translocates away from the NTD without CTD rearrangement, we do not observe characteristic ß-sheet-related FTIR signals in the $1640-1600 \mathrm{~cm}^{-1}$ region (Fig 4b). The EAS4 (infinity/steadystate) represents the CTD and NTD dissociation ${ }^{14}$ which is not observable in the crystallographic data due to the crystal packing constraints (Supplementary Discussion 7) ${ }^{2}$. The amide-I FTIR kinetics data and assignments agree well with our crystallographic observations, indicating that the same photoinduced structural rearrangements occur both in OCP solutions and in crystals.

This work provides the first structural basis for the initial reaction in OCP lightactivation. Our X-ray data demonstrates that the initial photoproduct is the trans-C7'-C8' cis photoisomerization of the CAN carotenoid chromophore. UV-VIS and IR spectroscopic studies in combination with harmonic frequency calculations confirm that the same reaction mechanism occurs in solution. The detailed description of the sequence of structural changes occurring upon illumination in response to the photoisomerization involves a propagation of structural changes from the NTD to the CTD. The presented evidence shows that the H-bond rupture between the carotenoid and the protein moiety does not initiate light activation but is a secondary event which follows post-photoisomerization NTD rearrangement.

\section{Acknowledgements}

The data were collected remotely at Diamond Light Source (BAG proposal number mx17221). 
This project was supported by the EMBO long-term fellowship (EMBO ALTF 244-2017) and has received funding from the European Union Horizon 2020 research and innovation programme under the Marie Sklodowska-Curie grant (agreement No. 839389) to V.C. This work was supported by the Leverhulme Trust award RPG-2018-372. The crystallisation facility at Imperial College was funded by the BBSRC (BB/D524840/1) and Wellcome Trust (202926/Z/16/Z). We thank Violeta Cordon Preciado for technical assistance.

\section{Author contributions}

V.U.C. designed the project. V.U.C. expressed and purified OCP protein, prepared OCP crystals, collected and processed X-ray data and UV-VIS/FTIR data. J.B assisted with creating Q-weighted maps and extrapolated coordinates. A.F. and J.J.vT. performed Harmonic Oscillator Calculations. R.M.M. assisted with cryo trapping experiment and X-ray data processing. M.A.P. assisted with the designing of OCP expression system in E.coli, while K.M. provided advice and support in obtaining OCP crystals. C.O. and A.W. performed long wavelength experiments. V.U.C. and J.J.vT. wrote the manuscript with the input from J.B., R.M.M., A.F., C.O. and A.W. J.B., R.M.M., A.F., C.O., A.W. and in particular K.M., V.U.C. and J.J.vT. contributed to the review and editing. J.J.vT. supervised the project.

\section{Competing interests}

The authors declare no competing interests.

\section{Data and materials availability}

Coordinates and structure factors for $\mathrm{OCP}_{\text {Dark }}$ and I1-I4 states in Fig. 1-3 will be made available via the PDB database.

\section{Correspondence}

Correspondence to Jasper van Thor. 
Figures

a

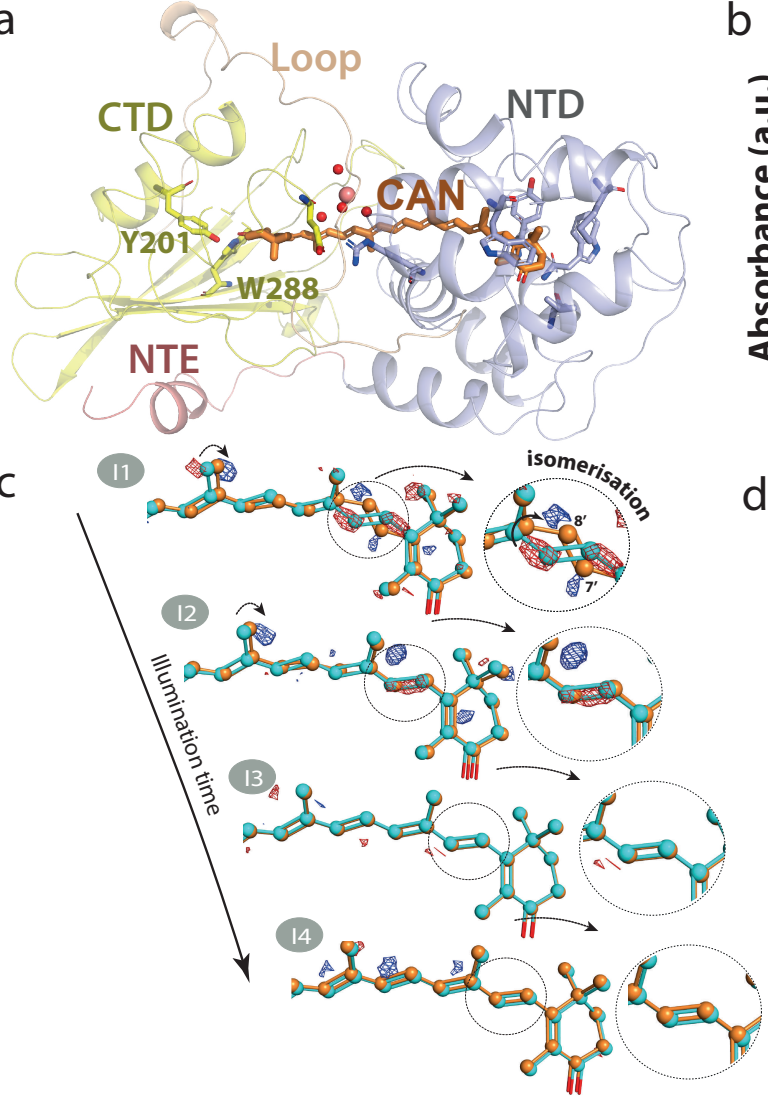

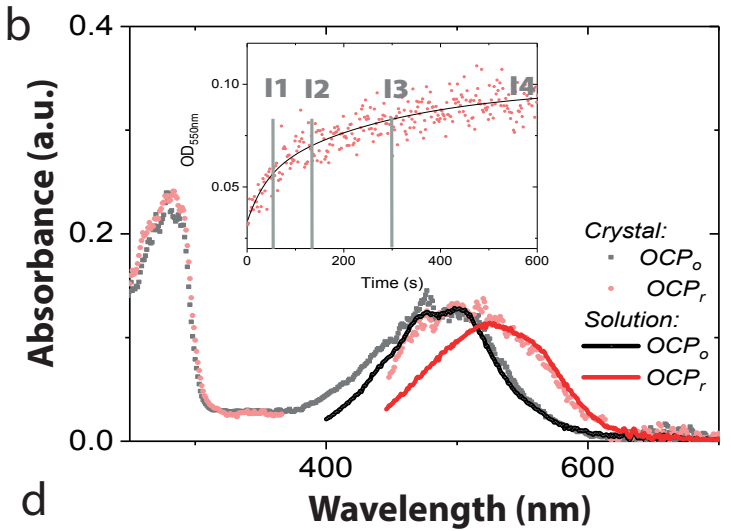

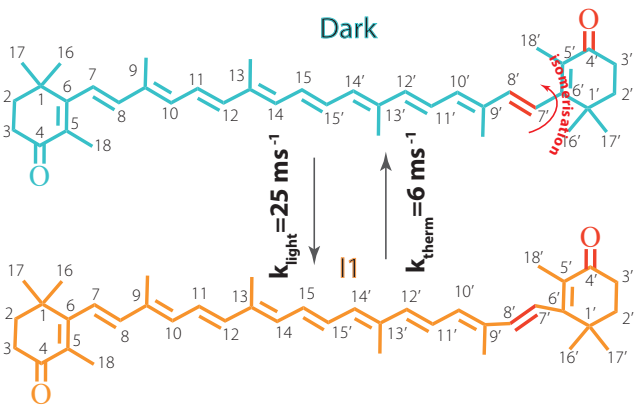

Fig. 1: Canthaxanthin C8'-C7' trans-to -cis isomerization triggers OCP light activation (a) Structure of OCP $_{\text {CAN }}$ in the 'dark' state displaying the C-terminal domain (CTD, yellow colour), N-terminal domain (NTD, blue colour), N-terminal extension (NTE, salmon colour) and loop (wheat colour). The canthaxanthin (CAN) carotenoid is embedded between the two domains. $\mathrm{Cl}^{-}$(pink sphere) is resolved in the CTD where it coordinates four water molecules (red spheres) through ion-dipole interactions at distances of 3.0-4.0 $\AA$. The closest CAN carbon (C12) is at $4.1 \AA$ distance (Supplementary Discussion 2). (b) Absorbance changes of OCP in solution (solid lines) and crystalline state (dotted lines) upon illumination. Inactive state $\mathrm{OCP}^{\mathrm{O}}$ and active state $\mathrm{OCP}^{\mathrm{R}}$ are shown in black/gray and red/pink respectively. Insert: Absorbance changes (pink dots) in the crystalline state detected at $550 \mathrm{~nm}$ upon $10 \mathrm{~min}$ of illumination with violet light (at $410 \mathrm{~nm}$ with $3.5 \mathrm{~mW} . \mathrm{cm}^{-2}$ power). Black line represents double exponential fit of the curve $\left(Y=A_{1} e^{x / t 1}+A_{2} e^{x / t 2}\right)$, where $A_{1}=0.02, t_{1}=38 \mathrm{~s}$ and $A_{2}=0.44, t_{2}=$ $291 \mathrm{~s}$. In comparison to solution measurements, the OCPR ${ }^{\mathrm{R}}$ spectrum maximum in crystals is blue-shifted by $5 \mathrm{~nm}$ and has slightly higher absorption in the 470-500 nm region probably due to conformational constraints imposed by the crystal packing which may prevent full separation of the CTD and NTD domains ${ }^{14}$.

(c) Difference electron density (DED) maps of CAN in $\mathrm{OCP}_{\text {Dark }}$, $\mathrm{OCP}_{\mathrm{I1}}, \mathrm{OCP}_{\mathrm{I} 2}, \mathrm{OCP}_{\mathrm{I} 3}$ and $\mathrm{OCP}_{\mathrm{I} 4}$ (orange mesh) (yellow/red at $\pm 3.0 \sigma$ ). $\mathrm{mFo}-\mathrm{DFc}$ of illuminated states are presented with green mesh (at $\pm 3.0 \sigma$ ). Difference electron density (DED) maps (blue/red at $\pm 3.5 \sigma$ ) were obtained for the four states: (I1) $1 \mathrm{~min}\left(\mathrm{~F}_{I 1}-\mathrm{F}_{\text {Dark }}\right.$ ), (I2) 2 $\min \left(\mathrm{F}_{12}-\mathrm{F}_{\text {Dark }}\right)$, (I3) $5 \mathrm{~min}\left(\mathrm{~F}_{13}-\mathrm{F}_{\text {Dark }}\right)$, (I4) $10 \mathrm{~min}\left(\mathrm{~F}_{14}-\mathrm{F}_{\text {Dark }}\right)$. Carotenoid coordinates of dark (OCP Dark) and light states $\left(\mathrm{OCP}_{11-14}\right)$ are represented by cyan and orange lines respectively. Arrow in the insert in 11 state indicates carotenoid movement during illumination. (d) All-trans CAN is present in OCPDark while C8'-C7' cis conformation is observed in $\mathrm{OCP}_{\mathrm{II}}$. The latter thermally recovers back to all-trans state with prolonged illumination time (after 2-5 min). 


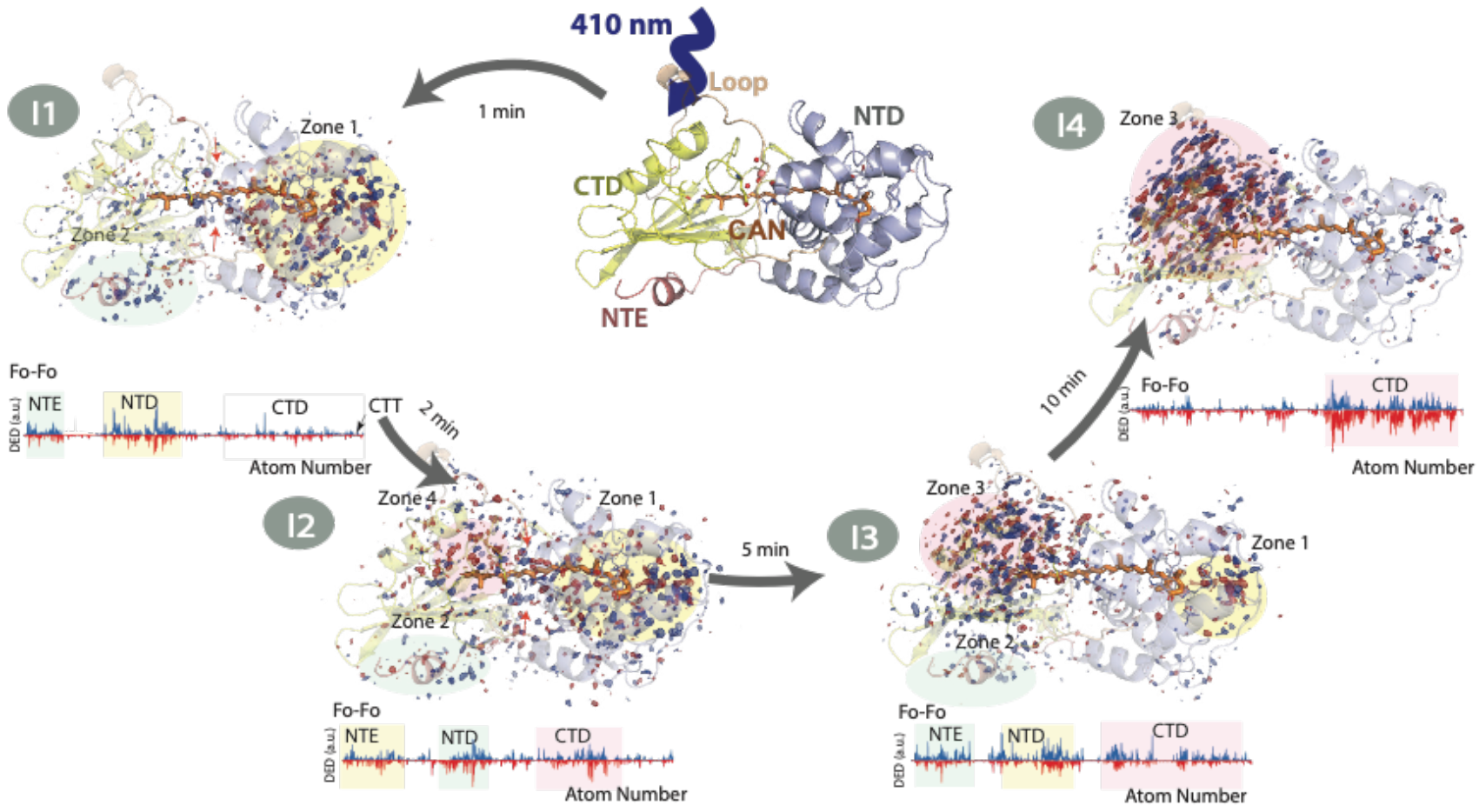

Fig. 2: Overall structural effect of illumination on OCP structure. Datasets from which all images are derived (Dark, I1-I4) have a resolution of 1.3-1.4 $\AA$ (Supplementary Table 5) and time points at which crystals were cryo-trapped are indicated for I1-I4. (I1- I4, upper panel) $\mathrm{F}_{0}-\mathrm{F}_{0}$ Difference electron density (DED) maps (blue/red at $\pm 3.5 \sigma$ ) for the four states shown below each corresponding structure: (I1) $1 \mathrm{~min}\left(\mathrm{~F}_{\mathrm{I} 1}-\mathrm{F}_{\mathrm{Dark}}\right)$, (I2) $2 \mathrm{~min}\left(\mathrm{~F}_{\mathrm{I} 2}-\mathrm{F}_{\text {Dark }}\right)$, (I3) $5 \mathrm{~min}\left(\mathrm{~F}_{\mathrm{I} 3}-\mathrm{F}_{\text {Dark }}\right)$ and (I4) 10 min ( $\left.\mathrm{F}_{\mathrm{I} 4}-\mathrm{F}_{\text {Dark }}\right)$. The DED signals are localised in the 3 major areas: zone 1: NTD; zone 2: NTE; zone 3: Alpha- helices in CTD. Note that some DED signals are observed at the interface between the two domains (red arrows in I1 and I2) while the C-terminal tail (CTT) does not exhibit any noticeable DED signal. See main text for explanations. (I1-I4, lower panel) DED signals are also shown as the sequential integrated signals around each atom in the protein chain. 


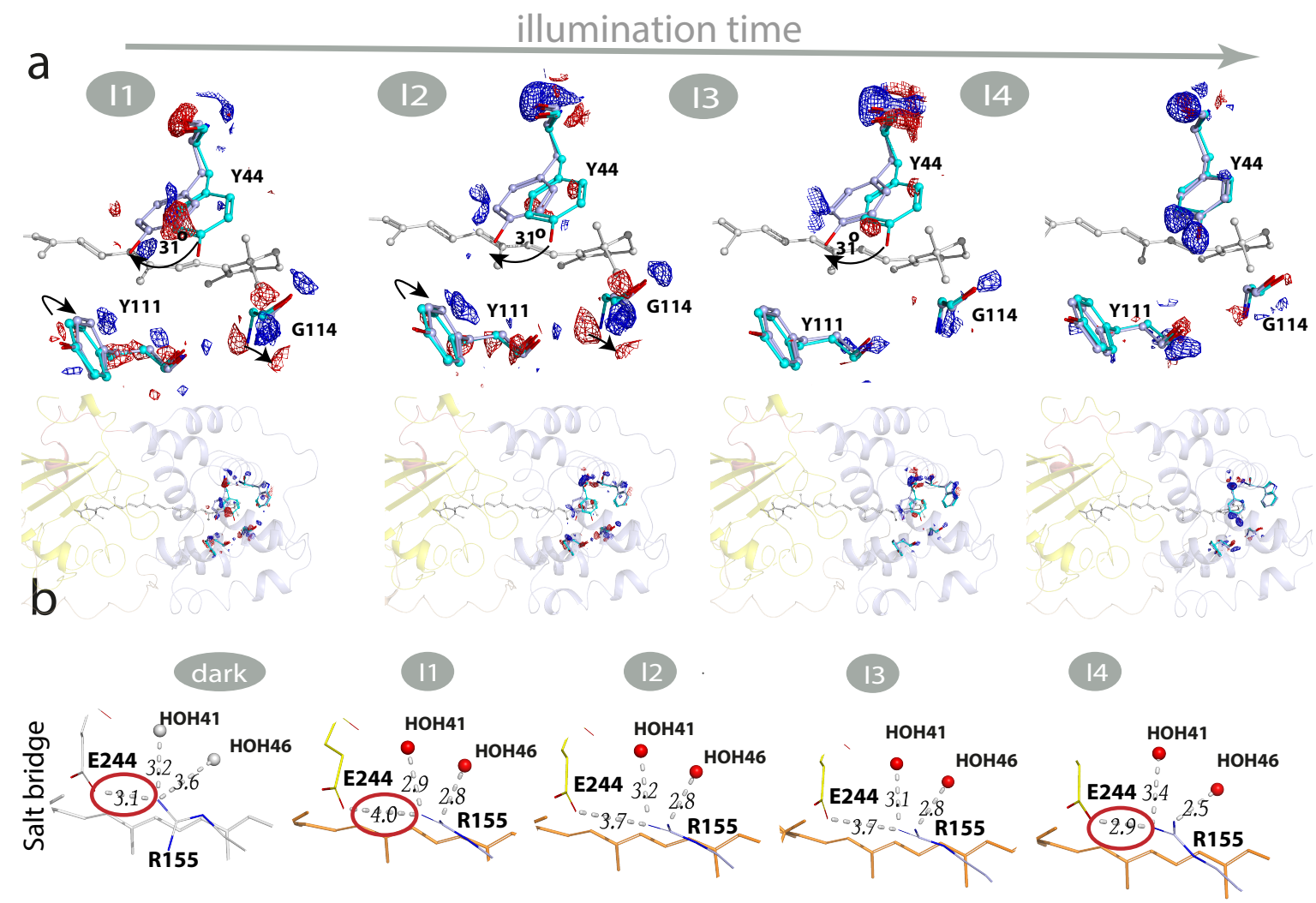

Fig. 3: OCP structural changes around C8'-C7' double bond in I1-I4 states upon illumination (a) Dark state coordinates are represented by cyan sticks while light state coordinates are represented by blue (NTD) and grey (CAN) sticks.). Difference electron density (DED) $F_{\text {Light }}-F_{\text {Dark }}$ are shown with blue/red mesh at $\pm 3.0 \sigma$ ). (b) Changes in the salt bridge formed between residues E244 and R155. 


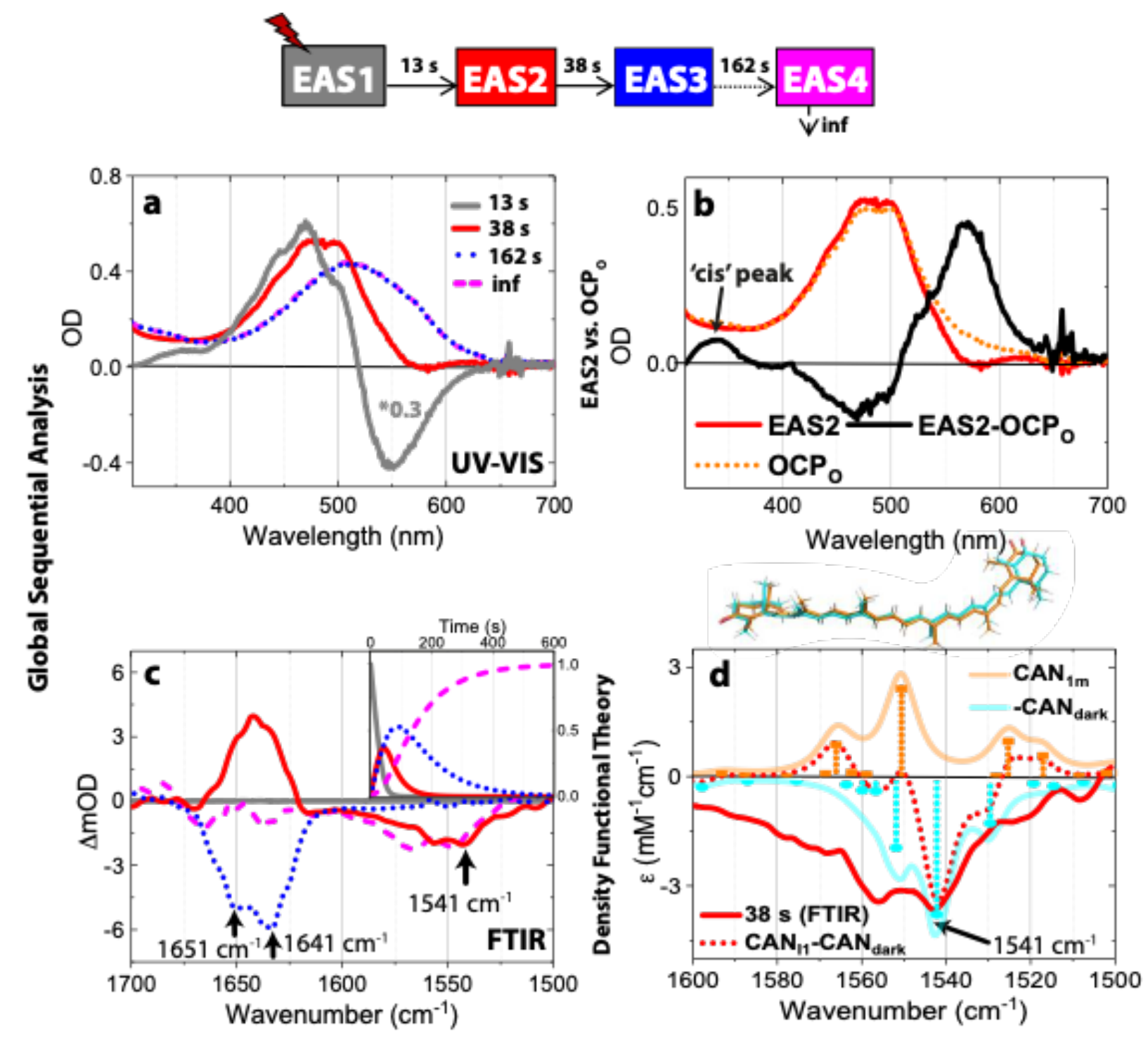

Fig. 4: Spectroscopic evidence for CAN C8'-C7' trans-to-cis isomerization in OCP in solution (a, c). Results of the simultaneous global fit of UV-VIS (a) and FTIR (c) data upon $10 \mathrm{~min}$ of illumination. The kinetic model used for the fit is shown on top of the panel with the respective lifetime for each transition between intermediates. The same colour code is used for the corresponding EAS components in panels a-b. Refer to the main text for an explanation of the relationship between the resolved EAS and the illuminated structural intermediates I1-I4 (Fig 1-3). (a) UV-VIS EAS. (b) EAS2 component was compared to the original $\mathrm{OCP}^{\mathrm{O}}$ spectrum before illumination at to. (c) FTIR EAS (in $\mathrm{D}_{2} \mathrm{O}$ ); FTIR (in $\mathrm{H}_{2} \mathrm{O}$ ) is shown in Supplementary Fig 16. Insert: Relative concentration profiles for each EAS. (d) Theoretical IR frequencies and intensities were calculated using Harmonic frequency calculations (HFC) at the B3LYP/6-311+G level. IR values were calculated for CAN in the two CAN Dark and $\mathrm{CAN}_{\mathrm{II}}$ states using $\mathrm{CAN}$ coordinates extracted from the extrapolated maps. Theoretical IR frequencies and calculated FTIR spectra are presented by sticks and solid lines respectively. Optimised CAN coordinates from HFC are shown on top of the panel. $\mathrm{CAN}_{\mathrm{II}}-\mathrm{CAN}_{\text {Dark }}$ (grey line) obtained from HFC agree with the $1542 \mathrm{~cm}^{-1}$ bleach from the 38 -s EAS2 (black line). 


\section{References}

1. Leverenz, R. L. et al. A $12 \AA$ carotenoid translocation in a photoswitch associated with cyanobacterial photoprotection. Science (80-. ). 348, 1463-1466 (2015).

2. Bandara, S. et al. Photoactivation mechanism of a carotenoid-based photoreceptor. Proc Natl Acad Sci U S A 114, 6286-6291 (2017).

3. Konold, P. E. et al. Photoactivation Mechanism, Timing of Protein Secondary Structure Dynamics and Carotenoid Translocation in the Orange Carotenoid Protein. $J$. Am. Chem. Soc. (2019). doi:10.1021/jacs.8b11373

4. Bondanza, M., Cupellini, L., Lipparini, F. \& Mennucci, B. The Multiple Roles of the Protein in the Photoactivation of Orange Carotenoid Protein. Chem 6, 187-203 (2020).

5. Maoka, T. Carotenoids as natural functional pigments. Journal of Natural Medicines (2020). doi:10.1007/s11418-019-01364-x

6. Polivka, T. \& Sundstrom, V. Ultrafast dynamics of carotenoid excited States-from solution to natural and artificial systems. Chem Rev 104, 2021-2071 (2004).

7. Polívka, T. \& Frank, H. A. Molecular factors controlling photosynthetic light harvesting by carotenoids. Acc. Chem. Res. (2010). doi:10.1021/ar100030m

8. Kromdijk, J. et al. Improving photosynthesis and crop productivity by accelerating recovery from photoprotection. Science (80-. ). (2016). doi:10.1126/science.aai8878

9. Ruban, A. V et al. Identification of a mechanism of photoprotective energy dissipation in higher plants. Nature 450, 575-578 (2007).

10. Liguori, N. et al. Different carotenoid conformations have distinct functions in lightharvesting regulation in plants. Nat. Commun. (2017). doi:10.1038/s41467-017-02239$\mathrm{Z}$

11. Pascal, A. A. et al. Molecular basis of photoprotection and control of photosynthetic light-harvesting. Nature 436, 134-137 (2005).

12. Muzzopappa, F. \& Kirilovsky, D. Changing Color for Photoprotection: The Orange Carotenoid Protein. Trends in Plant Science (2020). doi:10.1016/j.tplants.2019.09.013

13. Kerfeld, C. A. et al. The crystal structure of a cyanobacterial water-soluble carotenoid binding protein. Structure 11, 55-65 (2003).

14. Gupta, S. et al. Local and global structural drivers for the photoactivation of the orange carotenoid protein. Proc Natl Acad Sci U S A 112, E5567-74 (2015).

15. Kirilovsky, D. \& Kerfeld, C. A. The Orange Carotenoid Protein: A blue-green light photoactive protein. Photochem. Photobiol. Sci. 2, 1135-1143 (2013).

16. Wilson, A. et al. Structural determinants underlying photoprotection in the photoactive orange carotenoid protein of cyanobacteria. J. Biol. Chem. (2010).

doi:10.1074/jbc.M110.115709

17. Wilson, A. et al. The essential role of the N-terminal domain of the orange carotenoid protein in cyanobacterial photoprotection: Importance of a positive charge for phycobilisome binding. Plant Cell (2012). doi:10.1105/tpc.112.096909

18. Wilson, A., Punginelli, C., Couturier, M., Perreau, F. \& Kirilovsky, D. Essential role of two tyrosines and two tryptophans on the photoprotection activity of the Orange Carotenoid Protein. Biochim. Biophys. Acta-Bioenergetics 1807, 293-301 (2011).

19. Yaroshevich, I. A. et al. Role of hydrogen bond alternation and charge transfer states in photoactivation of the Orange Carotenoid Protein. Commun. Biol. (2021). doi:10.1038/s42003-021-02022-3

20. Wei, T., Balevičius, V., Polívka, T., Ruban, A. V.\& Duffy, C. D. P. How carotenoid distortions may determine optical properties: Lessons from the orange carotenoid protein. Phys. Chem. Chem. Phys. (2019). doi:10.1039/c9cp03574e

21. Nogly, P. et al. Retinal isomerization in bacteriorhodopsin captured by a femtosecond 
x-ray laser. Science (80-. ). (2018). doi:10.1126/science.aat0094

22. Gurchiek, J. K. et al. Fluorescence and Excited-State Conformational Dynamics of the Orange Carotenoid Protein. J. Phys. Chem. B (2018). doi:10.1021/acs.jpcb.7b09435

23. Ursby, T.\& Bourgeois, D. Improved Estimation of Structure-Factor Difference Amplitudes from Poorly Accurate Data. Acta Crystallogr. Sect. A Found. Crystallogr. (1997). doi:10.1107/S0108767397004522

24. Mezzetti, A. et al. Two-Step Structural Changes in Orange Carotenoid Protein Photoactivation Revealed by Time-Resolved Fourier Transform Infrared Spectroscopy. J. Phys. Chem. B (2019). doi:10.1021/acs.jpcb.9b01242

25. Zechmeister, L. \& Polgár, A. cis-trans Isomerization and Spectral Characteristics of Carotenoids and some Related Compounds. J. Am. Chem. Soc. (1943). doi:10.1021/ja01248a025

26. Polgár, A. \& Zechmeister, L. Action of Cold Concentrated Hydriodic Acid on Carotenes: Structure and cis-trans-Isomerization of Some Reaction Products. J. Am. Chem. Soc. (1943). doi:10.1021/ja01248a026

27. Philip, T. \& Francis, F. J. Isolation and chemical properties of capsanthin and derivatives. J. Food Sci. 36, 823-827 (1971).

28. Schlücker, S., Szeghalmi, A., Schmitt, M., Popp, J. \& Kiefer, W. Density functional and vibrational spectroscopic analysis of $\beta$-carotene. J. Raman Spectrosc. (2003). doi:10.1002/jrs.1013

29. Berezin, K. V.\& Nechaev, V. V. Calculation of the IR spectrum and the molecular structure of $\beta$-carotene. J. Appl. Spectrosc. (2005). doi:10.1007/s 10812-005-0049-x

30. Wilson, A. et al. A photoactive carotenoid protein acting as light intensity sensor. Proc. Natl. Acad. Sci. U. S. A. (2008). doi:10.1073/pnas.0804636105 